This item was submitted to Loughborough's Research Repository by the author.

Items in Figshare are protected by copyright, with all rights reserved, unless otherwise indicated.

\title{
Excitation of surface plasma waves across the layers of intrinsic Josephson junctions
}

\section{PLEASE CITE THE PUBLISHED VERSION}

http://dx.doi.org/10.1103/PhysRevB.80.184512

\section{PUBLISHER}

(C) American Physical Society

VERSION

VoR (Version of Record)

\section{LICENCE}

CC BY-NC-ND 4.0

\section{REPOSITORY RECORD}

Kadygrob, D.V., V.A. Golick, V.A. Yampol'skii, T.M. Slipchenko, Dmitry R. Gulevich, and Sergey Savel'ev. 2019. "Excitation of Surface Plasma Waves Across the Layers of Intrinsic Josephson Junctions". figshare. https://hdl.handle.net/2134/13127. 
This item was submitted to Loughborough's Institutional Repository (https://dspace.lboro.ac.uk/) by the author and is made available under the following Creative Commons Licence conditions.

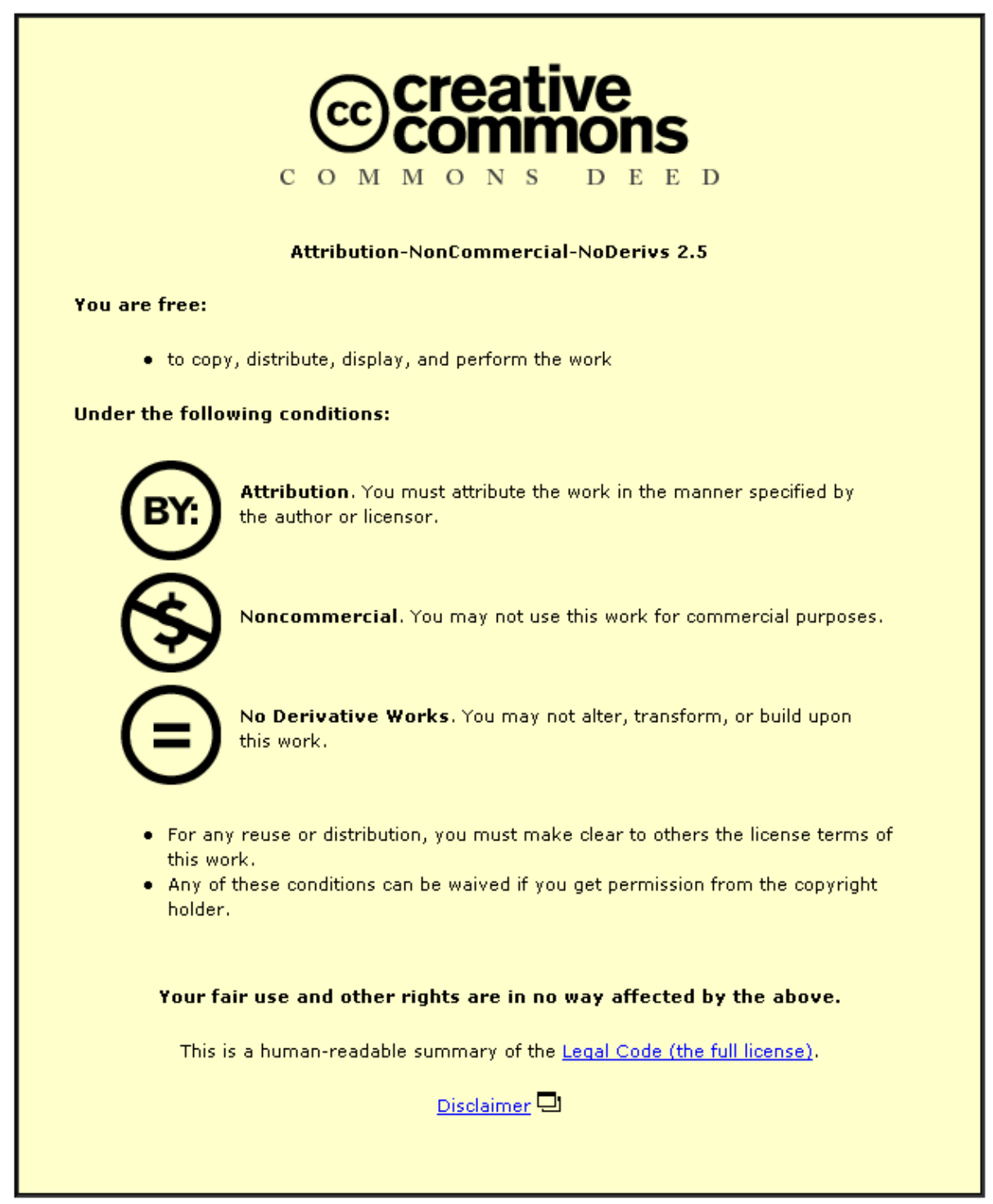

For the full text of this licence, please go to: http://creativecommons.org/licenses/by-nc-nd/2.5/ 


\title{
Excitation of surface plasma waves across the layers of intrinsic Josephson junctions
}

\author{
D. V. Kadygrob, ${ }^{1}$ V. A. Golick, ${ }^{2}$ V. A. Yampol'skii, ${ }^{1}$ T. M. Slipchenko, ${ }^{1}$ D. R. Gulevich, ${ }^{3}$ and Sergey Savel'ev ${ }^{3}$ \\ ${ }^{1}$ A.Ya. Usikov Institute for Radiophysics and Electronics, Ukrainian Academy of Sciences, 61085 Kharkov, Ukraine \\ ${ }^{2}$ V.N. Karazin Kharkov National University, 61077 Kharkov, Ukraine \\ ${ }^{3}$ Department of Physics, Loughborough University, Loughborough LE11 3TU, United Kingdom
}

(Received 20 August 2009; published 24 November 2009)

\begin{abstract}
We analytically study the excitation of surface Josephson plasma waves (SJPWs) propagating across the junctions in layered superconductors in the presence of external dc magnetic field. Both the attenuated total reflection and the modulation of the superconducting parameters methods of the SJPWs excitation are considered. We show that the reflection of the incident electromagnetic wave can be substantially decreased due to the resonance excitation of SJPWs, for certain angles and frequencies of the incident wave when changing the magnetic field. Moreover, we find physical conditions guaranteeing the total suppression of the specular reflectivity. The analytical results are supported by the numerical simulations.
\end{abstract}

DOI: $10.1103 /$ PhysRevB.80.184512

PACS number(s): 74.78.Fk, 74.50.+r, 74.72.Hs

\section{INTRODUCTION}

The physical properties of layered superconductors have attracted a great interest from many research groups. The strongly anisotropic high- $T_{c}$ single crystals are characteristic members of this family. The artificial stacks of Josephson junctions, e.g., $\mathrm{Nb}-\mathrm{Al}-\mathrm{AlO}_{x}-\mathrm{Nb}$, represent another group of such materials. Experiments for the c-axis conductivity in layered superconductors justify the use of a model, in which the superconducting $\mathrm{CuO}_{2}$ layers are coupled by the intrinsic Josephson effect through the layers. ${ }^{1-5}$ Thus, a very unusual plasma (the so-called Josephson plasma) is formed in layered superconductors. The current capability in this plasma is strongly anisotropic. Even the physical nature of the currents along and across the layers is principally different. Actually, the current along the layers is similar to the current in bulk superconductors, whereas the current across the layers has a Josephson origin.

The Josephson current flowing along the $\mathbf{c}$ axis couples with the electromagnetic field inside insulating dielectric layers, causing a specific kind of elementary excitations called the Josephson plasma waves (JPW) ${ }^{6-8}$ So, the layered superconducting structure favors propagation of electromagnetic waves through the layers.

Being of the similar origin as common plasma waves, JPWs propagate with frequencies above some threshold value (the Josephson plasma frequency $\omega_{J}$ ). The great challenge is to excite the electromagnetic waves in layered superconductors in a controllable manner because of their terahertz (THz) frequency range,, 10 which is still hardly reachable for both electronic and optical devices. Only recently the experimental evidence of excitation and emission of THz Josephson plasma waves has been reported. ${ }^{11}$

The presence of the sample boundary can produce a new branch of the wave spectrum below the Josephson plasma frequency, $\omega<\omega_{J}{ }^{12}$ i.e., surface Josephson plasma waves (SJPWs), which are analogous to the surface-plasmon polaritons in metals. ${ }^{13,14}$ Such waves can propagate in the vicinity of the superconducting surface along the layers. Later, the existence of surface electromagnetic waves propagating across the superconducting layers was predicted. ${ }^{15}$ In the lat- ter case, the spectrum of the surface waves is very sensitive to the external magnetic field. The profile of the amplitude oscillations of the electric-field component of such waves is peculiar: initially it increases toward the center of the superconductor and after reaching a crossover point decreases exponentially.

The dispersion curve, $\omega(q)$, of the surface waves lies below the "vacuum light line," $\omega=c q$, where $q$ is the wave number and $c$ is the speed of light. This means that the surface waves have wave vectors greater than the wave vectors of light of the same frequency in the vacuum. Thus, to excite the surface waves by means of incident electromagnetic waves it is necessary to use specific methods ${ }^{14}$ such as the attenuated total reflection (ATR) method or the modulation of the superconducting parameters (PM) method.

By means of the ATR method, one can excite a surface Josephson plasma wave by a wave incident from a dielectric prism onto a superconductor separated from the prism by a thin vacuum gap. In the absence of the superconductor, the incident wave completely reflects from the bottom of the prism if the incident angle $\theta$ exceeds the threshold angle $\theta_{t}$ for total internal reflection. However, the evanescent wave penetrates under the prism at a distance of about a vacuum wavelength. The wave vector of the evanescent mode is oriented along the bottom surface of the prism and its value is higher than $\omega / c$. This feature is the same as for surface waves. So, it is natural to expect the spatial matching and temporal matching (coincidence of both, the frequencies and wave vectors) of the evanescent modes and surface Josephson plasma waves for a certain incident angle. When the resonant excitation of SJPWs by the incident wave occurs, there is a strong suppression of the reflected wave.

The modulation of superconductor parameters can also result in the resonance excitation of SJPWs. The periodically modulated layered superconductor being irradiated by the electromagnetic wave with the tangential component $q$ of the wave vector, results in the excitation of diffracted waves (homogeneous and nonhomogeneous) with the wave vectors $q_{n}=q+n g$, where $g$ is the period of the reciprocal grating and $n$ is an integer. An SJPW would be excited if the tangential component of a wave vector of the $n$th diffracted wave coincides with the SJPW's wave vector, $\left|q_{n}\right|=\kappa_{\mathrm{sw}}$. Such a 


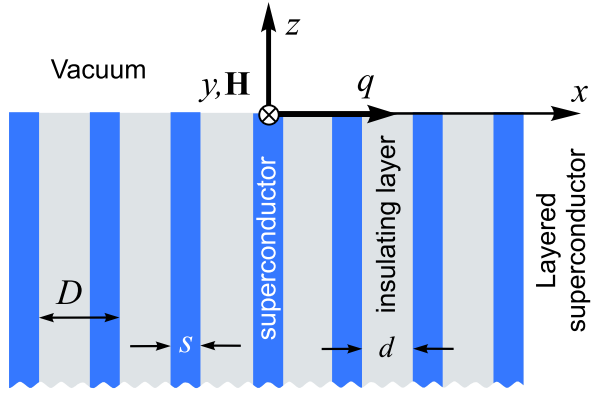

FIG. 1. (Color online) Geometry for studying surface waves across the layers.

matching can be reached by a proper choice of the frequency $\omega$ and/or the incident angle $\theta$. Under the resonance conditions, the amplitude of nonhomogeneous wave, which is responsible for the SJPW excitation, significantly grows and can exceed the amplitude of the incident wave. On the other hand, the SJPW excitation affects the amplitudes of other diffracted waves what leads to the strong suppression of the specularly reflected wave. Thus, changing $\omega$ and/or $\theta$, one can control the absorption and reflection of the electromagnetic wave. These phenomena are potentially useful for detecting $\mathrm{THz}$ radiation. The resonance phenomena described above are similar to the Wood anomalies in optics.

The problem of the SJPWs excitation along the layers by means of both the ATR and PM methods has been recently discussed. ${ }^{12}$ In the present work, we theoretically study the excitation of surface waves in layered superconductors across the layers. The ATR as well as PM methods of excitation of SJPWs are considered. One of the most important results is the prediction of the ability to control the Wood anomalies by means of an external magnetic field. In other words, the Wood anomalies can be observed for a certain incident angle $\theta$ and for a certain frequency $\omega$ of the incident wave when changing the magnetic field.

\section{MODEL}

Consider an interface (the $x y$ plane) separating the vacuum $(z>0)$ from a layered superconductor $(z \leq 0)$ (see Fig. 1). We study linear surface transverse magnetic (TM) electromagnetic waves propagating along the $x$ axis with the electric, $\mathbf{E}=\left\{E_{x}, 0, E_{z}\right\}$, and magnetic, $\mathbf{H}=\{0, H, 0\}$, components. The $x$ axis coincides with the crystallographic $\mathbf{c}$ axis. Thus, the electromagnetic wave propagates across the superconducting layers.

The electromagnetic field inside the layered superconductor is determined by the distribution of the gaugeinvariant phase difference $\varphi(x, z, t)$ of the order parameter between neighboring layers. It is described by a set of coupled sine-Gordon equations, ${ }^{16}$ which in the continuum limit (see, e.g., Ref. 17) can be written as

$$
\left(1-\lambda_{a b}^{2} \frac{\partial^{2}}{\partial x^{2}}\right)\left[\frac{\partial^{2} \varphi}{\partial t^{2}}+\omega_{r} \frac{\partial \varphi}{\partial t}+\omega_{J}^{2} \sin \varphi\right]-\lambda_{c}^{2} \omega_{J}^{2} \frac{\partial^{2} \varphi}{\partial z^{2}}=0 .
$$

Here $\omega_{r}=4 \pi \sigma_{c} / \varepsilon$ is the relaxation frequency proportional to the quasiparticle conductivity $\sigma_{c}$ across the layers, $\lambda_{a b}$ and $\lambda_{c}=c / \omega_{J} \sqrt{\varepsilon}$ are the magnetic penetration depths across and along layers, respectively, and $\omega_{J}=\left(8 \pi e D j_{c} / \hbar \varepsilon\right)^{1 / 2}$ is the Josephson plasma frequency. The latter is determined by the critical Josephson current density $j_{c}$, the interlayer dielectric constant $\varepsilon$, and the spatial period of the layered structure $D$.

As was shown in Ref. 18, the intralayer quasiparticle conductivity, $\sigma_{a b}$, should also be taken into account if $\omega$ is far enough from the Josephson plasma frequency. The contribution of the in-plane conductivity to the dissipation can be easily incorporated in our analysis. However, for the frequency range considered here (close to $\omega_{J}$ ), this contribution is strongly suppressed and can be safely omitted because the relative value of the term with $\sigma_{a b}$ is

$$
\left(\frac{\lambda_{a b}}{\lambda_{c}}\right)^{2}\left(\frac{\sigma_{a b}}{\sigma_{c}}\right)\left|1-\frac{\omega}{\omega_{J}}\right| \ll 1 .
$$

The electric and magnetic fields, $E^{s}$ and $H^{s}$, in the layered superconductor can be obtained from the distribution of the gauge-invariant phase difference using relations

$$
\begin{gathered}
E_{x}^{s}=\mathcal{H}_{0} \frac{1}{\omega_{J} \sqrt{\varepsilon}} \frac{\partial \varphi}{\partial t}, \quad E_{z}^{s}=\frac{\lambda_{a b}^{2}}{c} \frac{\partial^{2} H^{s}}{\partial t \partial x}, \\
-\frac{\partial H^{s}}{\partial z}=\frac{\mathcal{H}_{0}}{\lambda_{c}}\left[\frac{1}{\omega_{J}^{2}} \frac{\partial^{2} \varphi}{\partial t^{2}}+\frac{\omega_{r}}{\omega_{J}^{2}} \frac{\partial \varphi}{\partial t}+\sin \varphi\right], \\
-\frac{\partial \varphi}{\partial z}=\frac{2 \pi D}{\Phi_{0}}\left(1-\lambda_{a b}^{2} \frac{\partial^{2}}{\partial x^{2}}\right) H^{s},
\end{gathered}
$$

where $\mathcal{H}_{0}=\Phi_{0} / 2 \pi D \lambda_{c}, \Phi_{0}=\pi c \hbar / e$ is the flux quantum.

Note that the component $E_{x}$ of the electric field induces a charge in the superconducting layers when the charge compressibility is finite. This results in an additional interlayer coupling (the so-called capacitive coupling). ${ }^{19}$ Such a coupling significantly affects the properties of the longitudinal Josephson plasma waves with the wave vector perpendicular to the layers. The dispersion equation for the linear Josephson plasma waves with arbitrary direction of wave vector, and taking into account capacitive coupling, was obtained in Ref. 20. According to this dispersion equation, the capacitive coupling can be safely neglected in our case, when the wave vector has a component $k_{s} \gtrsim \omega / c$ along the layers, since $\alpha$ $=\varepsilon R_{D}^{2} / s D \ll 1$. Here $R_{D}$ is the Debye length for a charge in a superconductor and $s$ is the thickness of the superconducting layers. Below we consider the excitation of the electromagnetic field in a layered superconductor using the ATR and PM methods.

\section{A. Basic equations for the ATR method}

Here we study the excitation of the monochromatic SJPWs with field components proportional to $\exp [i(q x-\omega t)]$ and decaying into both the vacuum and layered superconductor away from the interface $z=0$. When $q>\omega / c$, the Maxwell equations yield an exponential decay of the wave amplitude into the vacuum

$$
H^{\mathrm{vac}}, E_{x}^{\mathrm{vac}}, E_{z}^{\mathrm{vac}} \propto \exp \left(i q x-i \omega t-k_{v} z\right), \quad z>0,
$$

with the decay constant 


$$
k_{v}=\sqrt{q^{2}-\frac{\omega^{2}}{c^{2}}}>0 .
$$

Moreover, the Maxwell equations provide the ratio of amplitudes for the tangential electric and magnetic fields at the interface $z=+0$ (i.e., right above the sample surface)

$$
\frac{E_{x}^{\mathrm{vac}}}{H^{\mathrm{vac}}}=\frac{i c}{\omega} k_{v}=\frac{i c}{\omega} \sqrt{q^{2}-\frac{\omega^{2}}{c^{2}}} .
$$

The linearized version of the coupled sine-Gordon Eq. (1), together with Eqs. (2)-(4), have a solution of the form

$$
\begin{gathered}
\varphi, H^{s}, E_{x}^{s} \propto \exp \left(i q x-i \omega t+k_{s} z\right), \\
E_{x}^{s}=-\mathcal{H}_{0} \frac{i \Omega}{\sqrt{\varepsilon}} \varphi, \\
H^{s}=-\frac{\mathcal{H}_{0}}{\lambda_{c} k_{s}}\left(1-\Omega^{2}-i \nu \Omega\right) \varphi,
\end{gathered}
$$

inside a layered superconductor and give the relation between the decay constant $k_{s}\left[\operatorname{Re}\left(k_{s}\right)>0\right]$, wave number $q$, and dimensionless frequency $\Omega=\omega / \omega_{J}$

$$
\left(1+\lambda_{a b}^{2} q^{2}\right)\left(1-\Omega^{2}-i \nu \Omega\right)=\lambda_{c}^{2} k_{s}^{2},
$$

where $\nu=\omega_{r} / \omega_{J}$ is the dissipation parameter.

The dispersion relation, $q(\omega)$, for the surface Josephson plasma wave can be obtained by matching the in-plane fields $H$ and $E_{x}$ at the vacuum-superconductor interface. Thus, in order to find the spectrum of the surface JPWs, we should derive the ratio $E_{x}^{s} / H^{s}$ at $z=0$ and use the impedance matching

$$
\frac{E_{x}^{\mathrm{vac}}}{H^{\mathrm{vac}}}=\frac{E_{x}^{s}}{H^{s}} .
$$

Thus, substituting Eqs. (6), (8), and (9) into Eq. (11) we obtain the dispersion relation for the surface Josephson plasma waves ${ }^{15}$

$$
\sqrt{Q^{2}-\frac{\lambda_{a b}^{2} \Omega^{2}}{\lambda_{c}^{2} \varepsilon}}=\frac{\lambda_{a b}^{2} \Omega^{2} \sqrt{1+Q^{2}}}{\lambda_{c} \varepsilon \sqrt{1-\Omega^{2}-i \nu \Omega}},
$$

with $Q=q \lambda_{a b}$. The dispersion curve for such waves is shown in Fig. 2(b).

One can excite an SJPW with the spectrum [Eq. (12)] by a wave incident from a dielectric prism onto a superconductor separated from the prism by a thin vacuum gap of thickness $\delta$ (see Fig. 3). The prism has permittivity $\epsilon$. The wave frequency $\omega$ is assumed to be below the Josephson plasma frequency $\omega_{J}$. The magnetic field $H^{d}$ in the dielectric prism can be represented as a sum of incident and reflected waves with amplitudes $H^{i}$ and $H^{r}$, respectively,

$$
\begin{gathered}
H^{d}=H^{i} \exp \left[i q x-i k_{d}(z-\delta)\right]+H^{r} \exp \left[i q x+i k_{d}(z-\delta)\right], \\
z>\delta .
\end{gathered}
$$

Here and below we omit the time-dependent multiplier, $\exp (-i \omega t)$. The plane $z=0$ corresponds to the vacuum-
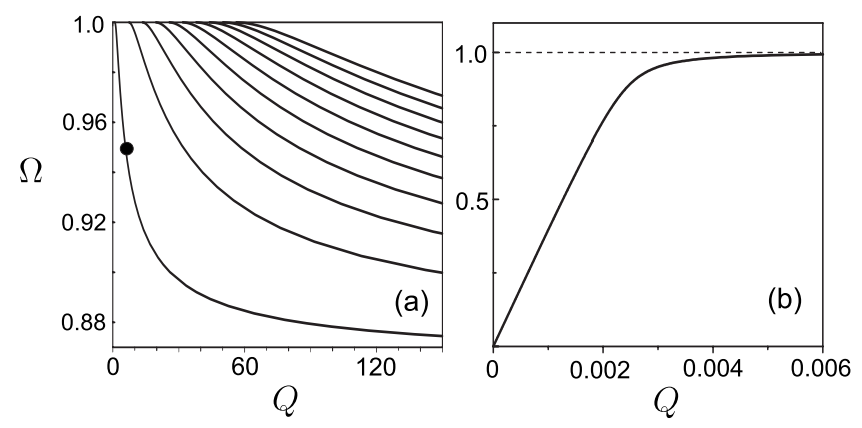

FIG. 2. The dispersion curves for the SJPWs across the layers. (a) The case of the SJPWs in the presence of the external magnetic field, $h_{0} / h_{c}=0.357$. The branches correspond to $n=0,1,2, \ldots, 9$ from bottom to top. The spectrum is limited from below by the value $\Omega>\left(1-2 h_{0}^{2} / h_{c}^{2}\right)^{1 / 2} \simeq 0.86$. The values of the parameters are: $\lambda_{c} / \lambda_{a b}=7, \varepsilon=4$. The solid circle on the line $n=0$ describes the SJPW simulated in Figs. 10 and 11. (b) The dispersion curve [Eq. (12)] for the SJPWs in the absence of the external magnetic field, $h_{0}=0 ; \lambda_{c} / \lambda_{a b}=100, \varepsilon=16$.

superconductor boundary. The tangential $q$ and normal $k_{d}$ components of the wave vector for waves in the prism are defined by

$$
q=k \sqrt{\epsilon} \sin \theta, \quad k_{d}=\sqrt{k^{2} \epsilon-q^{2}}=k \sqrt{\epsilon} \cos \theta,
$$

where $k=\omega / c$. The condition for total internal reflection of the wave in the dielectric prism is assumed to be fulfilled, i.e.,

$$
\sin ^{2} \theta>1 / \epsilon .
$$

The magnetic field

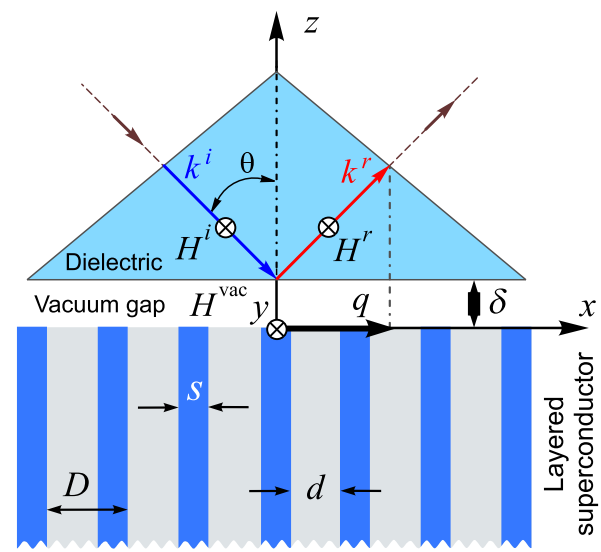

FIG. 3. (Color online) A dielectric prism is separated from a layered superconductor by a vacuum gap of thickness $\delta$. An electromagnetic wave with incident angle $\theta>\theta_{t}$ can excite SJPWs that satisfy the following resonance condition: $\omega \sqrt{\epsilon} \sin \theta / c=q$. Here $\mathbf{k}^{i}$ and $\mathbf{k}^{r}$ are the wave vectors of the incident and reflected waves associated with the magnetic-field amplitudes $\mathbf{H}^{i}$ and $\mathbf{H}^{r}$. The resonance excitation of SJPWs by the incident wave produces a strong suppression of the reflected wave. This method for producing surface waves is known as the ATR method. 


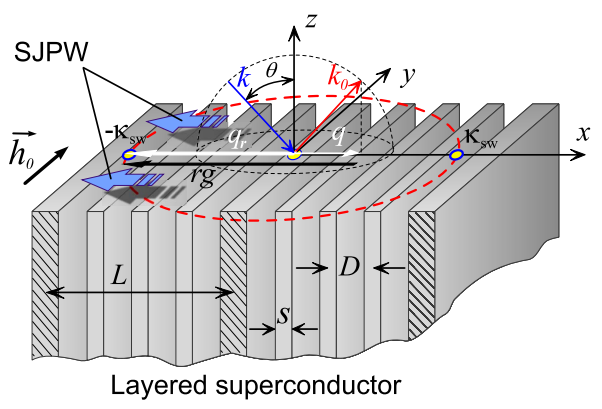

FIG. 4. (Color online) Geometry of the problem for the SJPW excitation by the PM method. The dashed layers are a bit thicker than others. $\mathbf{k}$ and $\mathbf{k}_{0}$ are the wave vectors of the incident and specularly reflected waves, $\kappa_{\mathrm{sW}}$ is the wave number of the SJPW. The case of backward resonance diffraction in the $r$ th order $(r<0)$ is shown. Also, $q_{r}=q+r g \simeq-\kappa_{\mathrm{sw}}$ denotes the tangential component of the wave vector of the resonance wave, and $g=2 \pi / L$ is the period of the reciprocal grating.

$$
H^{\mathrm{vac}}=H^{i}\left[h^{+} \exp \left(i q x+k_{v} z\right)+h^{-} \exp \left(i q x-k_{v} z\right)\right],
$$

of the evanescent mode in the vacuum gap is generated by the wave from the dielectric prism. Here $h^{+}\left(h^{-}\right)$are the dimensionless amplitudes of the evanescent waves that exponentially increase (decrease) with the spatial increment rate

$$
k_{v}=\sqrt{q^{2}-k^{2}}=k \sqrt{\epsilon \sin ^{2} \theta-1} .
$$

Using Maxwell's equations, one can express the $x$ components, $E_{x}^{d}$ and $E_{x}^{\mathrm{vac}}$, of the electric field in the dielectric prism and in the vacuum gap via the magnetic-field amplitudes

$$
\begin{gathered}
E_{x}^{d}=\frac{k_{d}}{k \epsilon} H^{i}\left\{h^{r} \exp \left[i q x+i k_{d}(z-\delta)\right]-\exp \left[i q x-i k_{d}(z-\delta)\right]\right\} \\
h^{r}=H^{r} / H^{i} \\
E_{x}^{\mathrm{vac}}=-i \frac{k_{v}}{k} H^{i}\left[h^{+} \exp \left(i q x+k_{v} z\right)-h^{-} \exp \left(i q x-k_{v} z\right)\right]
\end{gathered}
$$

\section{B. Basic equations for the PM method}

Surface Josephson plasma waves can be also excited by diffracting the electromagnetic wave incident onto the periodically modulated layered superconductor in the presence of the external magnetic field. The spectrum for such waves is described in Ref. 15.

Consider a semi-infinite layered superconductor in the geometry shown in Fig. 4. The crystallographic ab plane coincides with the $z y$ plane and the $\mathbf{c}$ axis is directed along the $x$ axis.

We suppose that the London penetration depth along the $x$ direction of the layered superconductor, $\lambda_{a b}=\lambda D /(s d)^{1 / 2}$, is periodically modulated in the $x$ direction with a spatial period $L$. Here $\lambda$ is the penetration depth for the bulk superconductor and $s \ll D$ is the thickness of a superconducting layer. Such a modulation can be realized, for instance, if we produce different thicknesses $s$ of superconducting layers. The Fourier expansion of $\lambda_{a b}^{2}(x)$ is

$$
\lambda_{a b}^{2}(x)=\lambda_{a b}^{2}\left[1+\sum_{n=-\infty}^{\infty} f_{n} \exp (\text { ing } x)\right],
$$

where $f_{-n}=f_{n}^{*}, f_{0}=0$, and $g=2 \pi / L$ is the period of the reciprocal grating.

Let an external magnetic field $\mathbf{h}_{0}$ be applied along the $y$ axis, parallel to the superconducting layers (see Fig. 4). We consider $h_{0}$ to be less than the critical value $h_{c}=\Phi_{0} / \pi D \lambda_{c}$, when the dc phase $\varphi_{0}(z)$ inside the superconductor coincides with the phase distribution in a "tail" of a Josephson vortex placed at a distance $z_{0}$ away from the boundary of the superconductor (see, e.g., Ref. 15)

$$
\begin{gathered}
\varphi_{0}(z)=4 \arctan \left[\exp \left(\frac{z-z_{0}}{\lambda_{c}}\right)\right], \quad z<0, \\
z_{0}=\lambda_{c} \operatorname{arccosh}\left(\frac{h_{c}}{h_{0}}\right) .
\end{gathered}
$$

Let a $p$-polarized (transverse magnetic) plane monochromatic electromagnetic wave with the electric, $\mathbf{E}=\left\{E_{x}, 0, E_{z}\right\}$, and magnetic, $\mathbf{H}=\{0, H, 0\}$, fields be incident onto the periodically modulated layered superconductor at an angle $\theta$ from the vacuum half-space. The in-plane and out-of-plane components of its wave vector are

$$
k_{x} \equiv q=k \sin \theta, \quad k_{z}=k \cos \theta .
$$

The in-plane periodic modulation results in generating the diffracted waves with in-plane and out-of-plane wave-vector components

$$
q_{n}=q+n g, \quad k_{z n}^{V}=\sqrt{k^{2}-q_{n}^{2}}, \quad \operatorname{Re}\left[k_{z n}^{V}\right], \quad \operatorname{Im}\left[k_{z n}^{V}\right] \geq 0,
$$

where $n$ is an integer and $g=2 \pi / L$. The magnetic field of the incident wave in the vacuum $(z>0)$ is given by the FourierFloquet expansion

$$
H^{V}(x, z)=H^{\mathrm{inc}}\left[\exp \left(i q x-i k_{z} z\right)+\sum_{n} R_{n} \exp \left(i q_{n} x+i k_{z n}^{V} z\right)\right]
$$

where $H^{\text {inc }}$ is the amplitude of the incident wave and $R_{n}$ are the transformation coefficients (TCs). Recall that the time dependence $\exp (-i \omega t)$ is omitted in all formulas. Using the Maxwell equations, we express the tangential component of the electric field in the vacuum in terms of the amplitudes of the magnetic field

$$
\begin{aligned}
E_{x}^{V}(x, z)= & H^{\mathrm{inc}}\left[-\beta^{V} \exp \left(i q x-i k_{z} z\right)\right. \\
& \left.+\sum_{n} \beta_{n}^{V} R_{n} \exp \left(i q_{n} x+i k_{z n}^{V} z\right)\right],
\end{aligned}
$$

where $\beta^{V}=\cos \theta, \beta_{n}^{V}=k_{z n}^{V} / k$.

The electromagnetic field inside the layered superconductor $(z<0)$ is determined by the distribution of the gaugeinvariant phase difference $\varphi(x, z, t)$. We consider the $t$ and $x$ dependence of the superconducting phase $\varphi(x, z, t)$ as small 
variations around the stationary configuration $\varphi_{0}(z)$ given by Eq. (21). Assuming

$$
\varphi(x, z, t)=\varphi_{0}(z)+\varphi_{w}(x, z, t),
$$

as a sum of the static and wave terms, we linearize Eq. (1) to obtain

$$
\begin{aligned}
{[1-} & \left.\lambda_{a b}^{2}(x) \frac{\partial^{2}}{\partial x^{2}}\right]\left[\frac{\partial^{2} \varphi_{w}}{\partial t^{2}}+\omega_{r} \frac{\partial \varphi_{w}}{\partial t}+\omega_{J}^{2} \varphi_{w} \cos \varphi_{0}(z)\right] \\
- & \lambda_{c}^{2} \omega_{J}^{2} \frac{\partial^{2} \varphi_{w}}{\partial z^{2}}=0 .
\end{aligned}
$$

Substituting $\varphi_{w}(x, z, t) \propto \exp \left(i q_{n} x-i \omega t\right)$ we derive an ordinary differential equation for $\varphi_{w}(x, z)$,

$$
\begin{gathered}
-\frac{\lambda_{c}^{2}}{1+q_{n}^{2} \lambda_{a b}^{2}(x)} \frac{d^{2} \varphi_{w}}{d z^{2}}+\left\{1-\Omega^{2}+i \nu \Omega\right. \\
\left.-\frac{2}{\cosh ^{2}\left[\left(z-z_{0}\right) / \lambda_{c}\right]}\right\} \varphi_{w}=0 .
\end{gathered}
$$

Here we are interested in a solution decaying inside the layered superconductor: $\varphi_{w}(x, z) \rightarrow 0$ at $z \rightarrow-\infty$. Equation (26) has the form of a one-dimensional Schrödinger equation for a particle with energy

$$
E(\Omega)=\Omega^{2}-1+i \nu \Omega
$$

in a reflectionless potential

$$
U(z)=-\frac{2}{\cosh ^{2}\left[\left(z-z_{0}\right) / \lambda_{c}\right]} .
$$

Inside the layered superconductor, we represent the gauge-invariant phase difference and the electromagnetic field as expansions over the eigenfunctions. One can write a solution of Eq. (25) in terms of the WKB approximation valid for $Q_{n}=q_{n} \lambda_{a b} \gg 1$. Taking into account Eqs. (2) and (3) we obtain

$$
\begin{gathered}
\varphi_{w}(x, z)=\sum_{n, s} K_{s} A_{n \mid s} \frac{\exp \left(i q_{n} x\right)}{[E-U(z)]^{1 / 4}} \cos \left[\eta_{s}(z)\right], \\
H^{s}(x, z)=H^{\mathrm{inc}} \sum_{n, s} K_{s} \Psi_{n \mid s} \frac{\exp \left(i q_{n} x\right)}{[E-U(z)]^{1 / 4}} \sin \left[\eta_{s}(z)\right], \\
E_{x}^{s}(x, z)=-H^{\mathrm{inc}} \sum_{n, s} a_{s} K_{s} \Psi_{n \mid s} \frac{\exp \left(i q_{n} x\right)}{[E-U(z)]^{1 / 4}} \sin \left[\eta_{s}(z)\right] .
\end{gathered}
$$

Here we introduce the dimensionless variable

$$
a_{s}(z)=\frac{i \Omega}{\sqrt{\varepsilon}} \frac{\sqrt{1+Q_{s}^{2}}}{\sqrt{E-U(z)}} \frac{1}{\tan \left[\eta_{s}(z)\right]},
$$

and the function

$$
\eta_{s}(z)=\left(\frac{\sqrt{1+Q_{s}^{2}}}{\lambda_{c}} \int_{z_{t}}^{z} d z^{\prime} \sqrt{E-U\left(z^{\prime}\right)}-\frac{\pi}{4}\right) .
$$

After substituting Eqs. (23), (24), (30), and (31) into Eq. (11), we get the following dispersion relation ${ }^{15}$

$$
\frac{\lambda_{a b} \Omega^{2}}{\lambda_{c} \varepsilon} \frac{\sqrt{1+Q^{2}}}{\sqrt{\left(Q^{2}-\frac{\lambda_{a b}^{2} \Omega^{2}}{\lambda_{c}^{2} \varepsilon}\right)\left[2 \frac{h_{0}^{2}}{h_{c}^{2}}+E(\Omega)\right]}}=\tan \left[\eta_{s}(0)\right] .
$$

with $Q=\kappa_{\mathrm{sw}} \lambda_{a b}, \kappa_{\mathrm{sw}}(\omega)>k$ is the SJPW wave number. Since $\lambda_{a b} / \lambda_{c} \varepsilon \ll 1$, this relation can be simplified disregarding the vacuum contribution

$$
\frac{\sqrt{1+Q^{2}}}{\lambda_{c}} \int_{z_{t}}^{0} d z^{\prime} \sqrt{E(\Omega)-U\left(z^{\prime}\right)}=\pi\left(n+\frac{1}{4}\right),
$$

with $n=0,1,2, \ldots$ A set of dispersion curves for $n$ $=0,1, \ldots, 9$ is shown in Fig. 2(a) for the external magnetic field $h_{0} / h_{c}=0.357$.

Below we show that the SJPWs with the spectrum [Eq. (35)] can be excited by the PM method. The resonance excitation of the SJPWs corresponds to the condition

$$
Q_{n}=(k \sin \theta+n g) \lambda_{a b}=\operatorname{sign}(n) \operatorname{Re}[Q(\Omega)],
$$

with $Q=\kappa_{\mathrm{sw}} \lambda_{a b}$.

The coefficients $A_{n \mid s}$ in Eq. (29) and $\Psi_{n \mid s}$ in Eqs. (30) and (31) can be found similar to Ref. 12. Substituting the expressions in Eqs. (29)-(31) into Eqs. (3) and (25) and excluding $A_{n \mid s}$, we derive the coefficients $\Psi_{n \mid s}$ using perturbations theory with respect to the small modulations, $\left|f_{n}\right| \ll 1$. In linear approximation and in the absence of the degeneracy of the corresponding matrix, i.e., at

$$
q_{n}^{2} \neq q_{s}^{2} \quad \text { for } n \neq s,
$$

we obtain

$$
\begin{gathered}
\Psi_{n \mid s}=\delta_{n, s}+\widetilde{\Psi}_{n \mid s}, \quad \widetilde{\Psi}_{n \mid n}=0, \\
\widetilde{\Psi}_{n \mid s} \simeq-\frac{Q_{s}^{2}}{Q_{n}^{2}-Q_{s}^{2}} f_{n-s}, \quad n \neq s,
\end{gathered}
$$

where $\delta_{n, s}$ is the Kronecker delta.

\section{RESULTS}

In this section, we describe the results obtained on the basis of the model discussed above. We derive expressions for the reflectivity coefficients using both the ATR and PM methods. Moreover, we show that choosing a certain set of parameters one can completely suppress the reflection of the incident wave.

\section{A. Suppression of the specular reflectivity using the ATR method}

Using the conditions of continuity of the magnetic field and of the tangential components of the electric field at the 
dielectric-vacuum and vacuum-layered superconductor interfaces (given in Sec. II A), one obtains a set of four linear algebraic equations for four unknown wave amplitudes, $h^{r}, h^{+}, h^{-}$, and $H_{0}^{s}$ [here $H_{0}^{s}$ is the amplitude of the magnetic field $H^{s}$ in Eq. (7)]. Solving this set gives the reflection coefficient

$$
R \equiv h^{r}=\frac{R_{F}\left(k_{v} / k-a\right)+\left(k_{v} / k+a\right) C(\delta, \theta)}{\left(k_{v} / k-a\right)+\left(k_{v} / k+a\right) R_{F} C(\delta, \theta)}
$$

for the wave reflected from the bottom of the prism. Here

$$
R_{F}=\frac{k_{d}-i k_{v} \epsilon}{k_{d}+i k_{v} \epsilon} \equiv \exp (-i \psi)
$$

is the Fresnel reflection coefficient

$$
C(\delta, \theta)=\exp \left(-2 k_{v} \delta\right)
$$

is the parameter that provides the coupling between waves in the dielectric prism and the layered superconductor. Also

$$
a \equiv a(\Omega, \theta)=\frac{\Omega}{\sqrt{\varepsilon}}\left(\frac{1+Q^{2}}{1-\Omega^{2}-i \nu \Omega}\right)^{1 / 2}
$$

is the effective surface impedance of the superconductor.

Below we assume the coupling parameter $C$ to be small. However, even when $C \ll 1$, the coupling of the waves in the dielectric prism and superconductor plays a very important role in the excitation of SJPWs. First, the dispersion relation for the surface Josephson plasma waves is modified, involving a radiation leakage through the dielectric prism. The new spectrum of the SJPWs is defined by the denominator in Eq. (39). Actually, the region where the coupling $C \ll 1$ (when the radiation leakage of the excited SJPW through the prism does not dominate) corresponds to the strongest excitation of the surface waves by the incident wave. Furthermore, the coupling results in breaking the total internal reflection of the electromagnetic waves from the dielectric-vacuum interface. Due to this coupling, the reflection coefficient $R$ in Eq. (39) differs from the Fresnel one, $R_{F}$, its modulus becoming less than unity. Moreover, as we show below, the reflection of waves with any frequency $\omega<\omega_{J}$ can be completely suppressed for the specific incident angle $\theta$ and depth $\delta$ of the vacuum gap. This provides a way to control, detect, and filter $\mathrm{THz}$ radiation. Below we assume the dissipation parameter $\nu$ to be small compared to $\left(1-\Omega^{2}\right)$,

$$
\nu \ll 1-\Omega^{2} \text {. }
$$

Then the complex parameter $a(\Omega, \theta)$ in Eq. (42) can be presented as

$$
a(\Omega, \theta) \equiv a^{\prime}+i a^{\prime \prime}=\frac{\Omega}{\sqrt{\varepsilon}}\left(\frac{1+Q^{2}}{1-\Omega^{2}}\right)^{1 / 2}\left(1+\frac{1}{2} \frac{i \nu \Omega}{1-\Omega^{2}}\right) .
$$

Taking into account that $k_{v} / k \cong a^{\prime}$ in the vicinity of the SJPW spectrum, we can rewrite the reflection coefficient $R$ in the form

$$
R=R_{F} \frac{X(\Omega, \theta)-i B(\Omega, \theta)\left[C_{\mathrm{opt}}(\Omega, \theta)-C(\delta, \theta)\right]}{X(\Omega, \theta)-i B(\Omega, \theta)\left[C_{\mathrm{opt}}(\Omega, \theta)+C(\delta, \theta)\right]},
$$

with

$$
\begin{gathered}
X(\Omega, \theta)=\frac{k_{v}}{k}+a^{\prime}(2 C \cos \psi-1), \\
B(\Omega, \theta)=2 a^{\prime} \sin \psi,
\end{gathered}
$$

$$
C_{\text {opt }}(\Omega, \theta)=\frac{a^{\prime \prime}}{2 a^{\prime} \sin \psi},
$$

where $k_{v} \equiv k_{v}(\theta), a^{\prime} \equiv a^{\prime}(\Omega, \theta)$, and $a^{\prime \prime} \equiv a^{\prime \prime}(\Omega, \theta)$. Besides, the relation between $\psi, a^{\prime}(\Omega, \theta)$, and $\theta$ takes the form

$$
\tan \psi=\frac{2 a^{\prime} \sqrt{\epsilon} \cos \theta}{\cos ^{2} \theta-a^{\prime 2} \epsilon} .
$$

Equations (45) and (46) show that the modulus of the reflectivity $R(\theta)$ has a sharp resonance minimum at

$$
\sin \theta=\sin \theta_{\text {res }}=\left\{\frac{1+(\varepsilon-1)\left(1-\Omega^{2}\right)}{\epsilon\left[\varepsilon\left(1-\Omega^{2}\right)-\lambda_{a b}^{2} \Omega^{2} k^{2}\right]}\right\}^{1 / 2} .
$$

Since $\sin \theta_{\text {res }}<1$, the resonance can be observed only for

$$
1-\Omega^{2}>\frac{1}{\varepsilon(\epsilon-1)} .
$$

The minimum value of $R$ is

$$
|R|_{\text {min }} \cong \frac{\left|C_{\mathrm{opt}}\left(\Omega, \theta_{\mathrm{res}}\right)-C\left(\delta, \theta_{\mathrm{res}}\right)\right|}{C_{\mathrm{opt}}\left(\Omega, \theta_{\mathrm{res}}\right)+C\left(\delta, \theta_{\mathrm{res}}\right)} .
$$

This value strongly depends on the frequency detuning $(1-\Omega)$, dissipation parameter $\nu$, and the coupling between the waves in the dielectric prism and the layered superconductor, i.e., on the thickness $\delta$ of the vacuum gap. This offers several important applications of the predicted anomaly in the reflectivity in the $\mathrm{THz}$ range. For instance, if the coupling parameter $C\left(\delta, \theta_{\text {res }}\right)$ is equal to the optimal value $C_{\text {opt }}$, i.e., the thickness, $\delta$ takes the optimal value

$$
\begin{aligned}
\delta_{\mathrm{opt}}= & \left.\frac{1}{2 k_{v}} \ln \frac{4 \sin \psi\left(1-\Omega^{2}\right)}{\nu \Omega}\right|_{\theta=\theta_{\mathrm{res}}} \\
= & \frac{1}{2 k \sqrt{\epsilon \sin ^{2} \theta_{\mathrm{res}}-1}} \\
& \times \ln \left\{\frac{8 \sqrt{\epsilon\left(\epsilon \sin ^{2} \theta_{\mathrm{res}}-1\right)} \cos \theta_{\mathrm{res}}\left(1-\Omega^{2}\right)}{\left[\epsilon\left(\epsilon \sin ^{2} \theta_{\mathrm{res}}-1\right)+\cos ^{2} \theta_{\mathrm{res}}\right] \nu \Omega}\right\},
\end{aligned}
$$

the reflection coefficient $R$ at $\theta=\theta_{\text {res }}$ vanishes. This means that a complete suppression of the reflectivity can be achieved by an appropriate choice of the parameters due to the resonant excitation of the surface Josephson plasma waves.

Figure 5 shows the resonance suppression of the reflectivity. Figure 6 demonstrates the sharp decrease in the reflectivity in the $(\theta, \Omega)$ plane due to the resonant excitation of the 


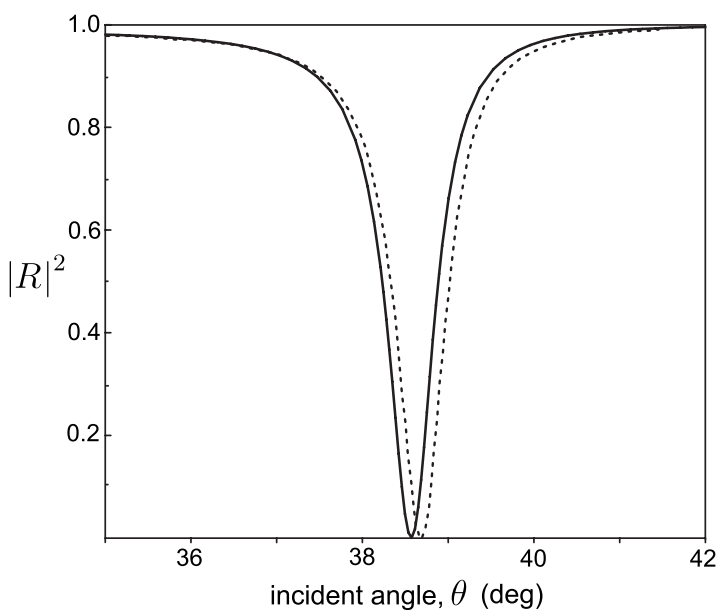

FIG. 5. The dependence of the reflectivity coefficient $|R|^{2}$ on the incident angle $\theta$ that presents the numerical calculations using Eqs. (44)-(48) (solid curve) and Eq. (39) (dotted curve) for parameters $\nu=2 \times 10^{-3}, \lambda_{a b} k=3 \times 10^{-3},\left(1-\Omega^{2}\right)=0.1, \varepsilon=16$, and $\epsilon=4$. The thickness of the vacuum gap is $\delta=\pi / k$. In this case, the limiting angle for the total internal reflection is $\theta_{t}=30^{\circ}$.

surface Josephson plasma waves. Obviously, the suppression of the reflectivity can be observed by changing the frequency at a given incident angle, as is shown in Fig. 7.

We also illustrated the effect of total suppression of the specular reflection by the distribution of the total magnetic field in the vacuum and the dielectric prism (Figs. 8 and 9). The interference pattern is seen for the nonresonant case (see Fig. 8), when the amplitudes of the incident and reflected waves practically coincide. Under the resonance condition (see Fig. 9), when the reflected wave is totally suppressed, the interference pattern in the far field disappears, while the near-field "torch" structure of the SJPW is clearly seen near the vacuum-layered superconductor interface.

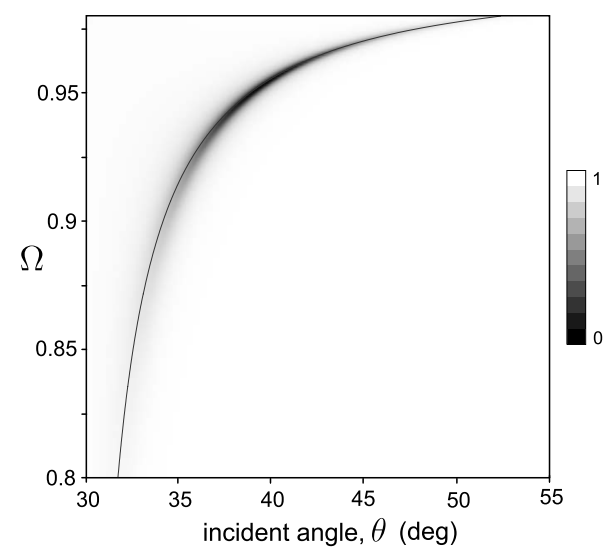

FIG. 6. The reflectivity coefficient in the plane $(\theta, \Omega)$ shown in gray levels. Other parameters are: $\nu=2 \times 10^{-3}, \varepsilon=16$, and $\epsilon=4$, $\lambda_{c} / \lambda_{a b}=100$. The thickness of the vacuum gap is $\delta=\pi / k$. The dispersion relation for the wave in the dielectric-vacuum-layered superconductor system is presented by the solid curve.

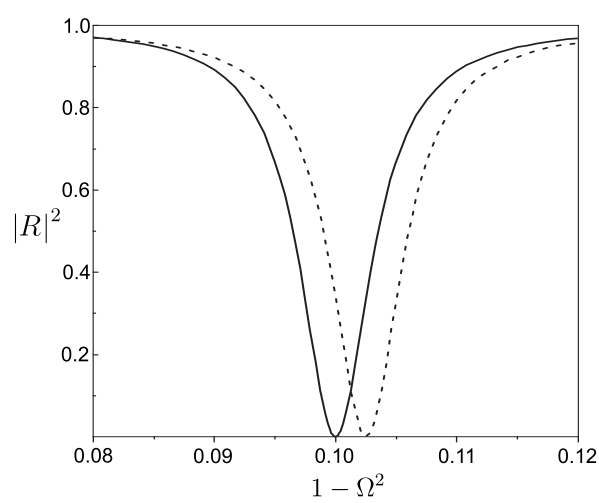

FIG. 7. The dependence of the reflectivity coefficient $|R|^{2}$ on $\left(1-\Omega^{2}\right)$, obtained for the incident angle $38.7^{\circ}$ using Eqs. (44)-(48) (solid curve) and Eq. (39) (dotted curve) for the same parameters as in Fig. 5.

\section{B. Suppression of the specular reflectivity using the PM method}

The boundary conditions, i.e., the continuity of the electric and magnetic-field components parallel to the surface, allow us to derive the transformation coefficients. Matching fields of Eqs. (23) and (30) with Eqs. (24) and (31) at the vacuum-superconductor interface, i.e., at $z=0$, we obtain

$$
\begin{aligned}
& \exp (i q x)+\sum_{n} R_{n} \exp \left(i q_{n} x\right) \\
& =\sum_{n, s} K_{s} \Psi_{n \mid s} \frac{\exp \left(i q_{n} x\right)}{\left[2\left(h_{0} / h_{c}\right)^{2}+E(\Omega)\right]^{1 / 4}} \sin \left[\eta_{s}(0)\right],
\end{aligned}
$$

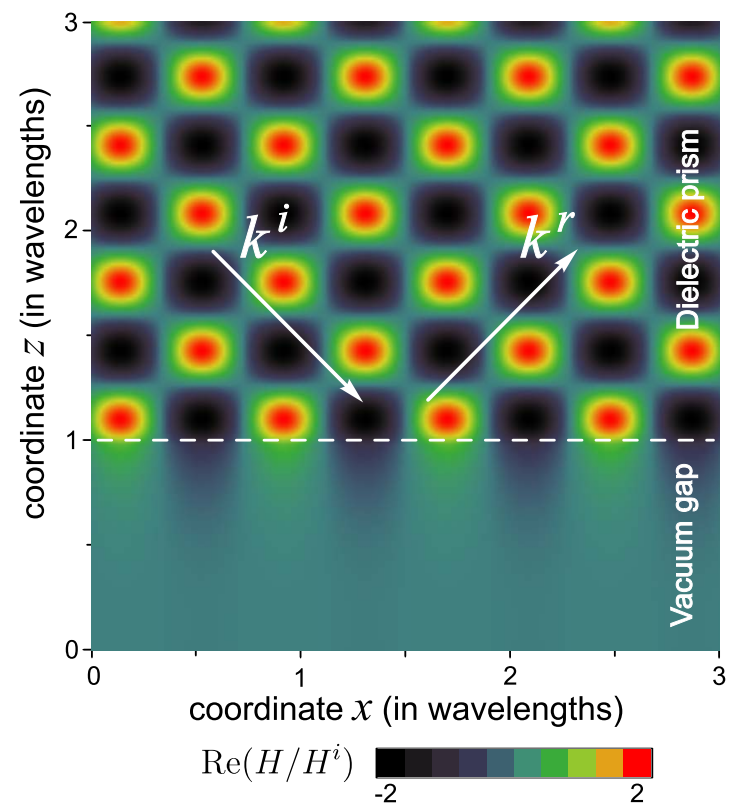

FIG. 8. (Color online) The magnetic-field distribution for the nonresonant case, $\theta=40^{\circ}$. Other parameters are: $\nu=2 \times 10^{-3}, \Omega$ $=0.89, \varepsilon=16, \epsilon=4$, and $\lambda_{c} / \lambda_{a b}=200$. The thickness of the vacuum gap is $\delta=2 \pi / k$. 


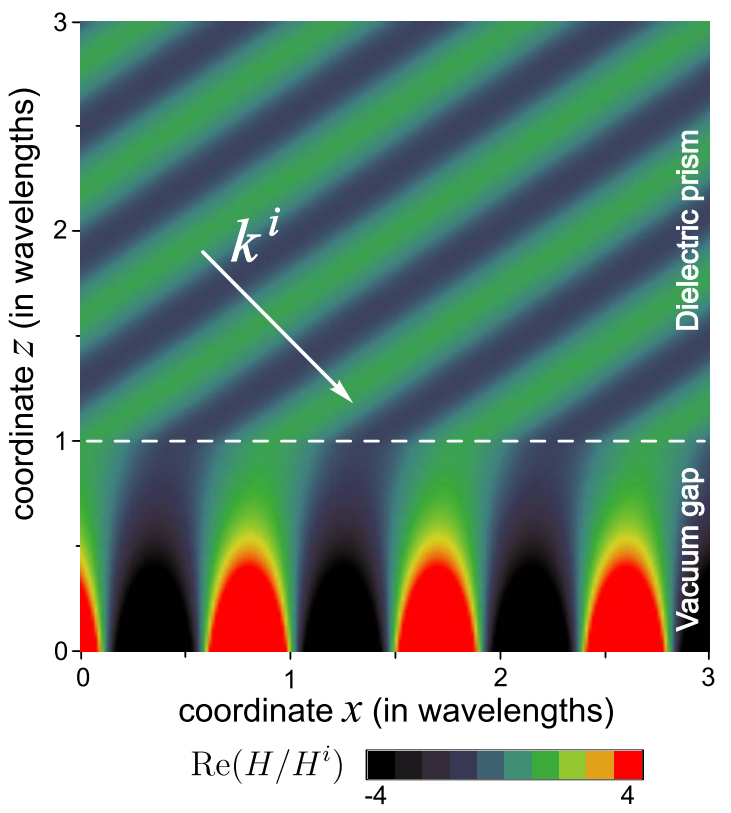

FIG. 9. (Color online) The magnetic-field distribution for the resonant case, $\theta=\theta_{\text {res }}=33.8^{\circ}$. Other parameters are the same as in Fig. 8.

$$
\begin{aligned}
\beta^{V} & \exp (i q x)-\sum_{n} \beta_{n}^{V} R_{n} \exp \left(i q_{n} x\right) \\
& =\sum_{n, s} a_{s} K_{s} \Psi_{n \mid s} \frac{\exp \left(i q_{n} x\right)}{\left[2\left(h_{0} / h_{c}\right)^{2}+E(\Omega)\right]^{1 / 4}} \sin \left[\eta_{s}(0)\right],
\end{aligned}
$$

where $a_{s} \equiv a_{s}(0)$. Equation (54) yields an infinite set of linear algebraic equations for the coefficients

$$
B_{s}=\frac{K_{s} \sin \left[\eta_{s}(0)\right]}{\left[2\left(h_{0} / h_{c}\right)^{2}+E(\Omega)\right]^{1 / 4}}
$$

and their relations to the TCs $R_{n}$,

$$
\begin{gathered}
\sum_{s} D_{n \mid s} B_{s}=2 \beta^{V} \delta_{n, 0}, \\
R_{n}=\sum_{s} B_{s} \Psi_{n \mid s}-\delta_{n, 0},
\end{gathered}
$$

where

$$
\begin{gathered}
D_{n \mid s}=b_{n} \delta_{n, s}+d_{n \mid s}, \quad b_{n}=\beta_{n}^{V}+a_{n}, \\
d_{n \mid s}=\left(\beta_{n}^{V}+a_{s}\right) \tilde{\Psi}_{n \mid s} .
\end{gathered}
$$

To solve the infinite set of Eq. (56) for $B_{s}$ we use resonant perturbation theory, which allows to present results in an explicit analytical form. ${ }^{21}$

When all spatial field harmonics are far away from the eigenmodes of the unmodulated layered superconductor (nonresonance conditions), the diagonal elements $b_{s}$ of the matrix $\hat{D} \equiv\left\|D_{n \mid s}\right\|$ are on the order of one or larger, $\left|b_{s}\right|$ $\sim\left|\beta_{s}^{V}\right| \geqslant 1$. In this case, the matrix $\hat{D}$ is diagonal dominated, that is, its off-diagonal elements are small compared to the diagonal ones, $\left|d_{n \mid s}\right| \sim\left|f_{n-s}\right| \ll\left|b_{s}\right|$. Then, the solution of Eqs.
(56) and (57) gives us a trivial result: the specular reflection $\mathrm{TC}, R_{0}$, is close to the Fresnel coefficient

$$
R_{F}=\frac{\cos \theta-a_{0}}{\cos \theta+a_{0}}
$$

and differs from it by terms proportional to $f^{2}$. Other TCs are small, $R_{n} \sim f_{n}, n \neq 0$. A much more interesting case occurs under the resonance conditions, when Eq. (36) holds for one (or simultaneously for two) spatial field (resonance) harmonics

$$
Q_{r}=(k \sin \theta+r g) \lambda_{a b} \simeq \operatorname{sign}(r) \operatorname{Re}[Q(\Omega)] .
$$

Here $r>0(r<0)$ corresponds to the forward (backward) propagation of the excited SJPWs with respect to the incident wave.

For simplicity, we restrict ourselves to the singleresonance case. Under the resonance conditions, the diagonal matrix element $D_{r \mid r}=b_{r}$ becomes anomalously small, and the determinant of the matrix $\hat{D}$ decreases significantly (see, e.g., Ref. 21). Recall that the normalized $z$ components of the wave vectors in the vacuum, $\beta_{s}^{V}$, can be either purely real or purely imaginary. Therefore, the minimum of $\left|b_{r}\right| \ll 1$ holds in the vicinity of the point in the $(\omega, \theta)$ plane, where $\operatorname{Im}\left[\beta_{r}^{V}\right]=-\operatorname{Im}\left[a_{r}\right]$, which is the dispersion relation for SJPWs, Eq. (34). According to Refs. 12 and 21, we come to the following relation for the specular TC, $R_{0}$,

$$
R_{0}=R_{F} \frac{k_{z r}^{V} / k+a_{r}+C_{r}\left(\theta, \Omega, f_{r}\right)-\Delta_{r}\left(\theta, \Omega, f_{r}\right)}{k_{z r}^{V} / k+a_{r}+C_{r}\left(\theta, \Omega, f_{r}\right)},
$$

where

$$
\Delta_{r}\left(\theta, \Omega, f_{r}\right)=\frac{2 \cos \theta}{\cos ^{2} \theta-a_{0}^{2}} \times\left(a_{0}-a_{r}\right)\left(k_{z r}^{V} / k+a_{0}\right) \widetilde{\Psi}_{0 \mid r} \tilde{\Psi}_{r \mid 0}
$$

and

$$
C_{r}=-\sum_{N} \frac{d_{r \mid N} d_{N \mid r}}{b_{N}}
$$

$C_{r} \equiv C_{r}\left(\theta, \Omega, f_{r}\right)$ is the parameter that describes the coupling between waves in the vacuum and layered superconductor. In the following numerical calculations for the specular coefficient $R_{0}$ we consider $C_{r}$ to be small. However, even when $\left|C_{r}\right| \ll 1$, the coupling of the waves in the vacuum and superconductor plays a very important role in the excitation of SJPWs and in the anomalies of the reflection properties (similar to the case of the ATR method of the SJPWs excitation).

First, the dispersion relation for the surface Josephson plasma waves is modified, involving a radiation leakage in the vacuum. The new spectrum of the SJPWs is defined by equating the denominator in Eq. (62) to zero. Thus, the quadratic in the modulation term, $C_{r}$ [see Eq. (64)], is responsible for the shift in the position of the resonance, $\operatorname{Im}\left[C_{r}\right]$, and its widening, $\operatorname{Re}\left[C_{r}\right]$. The region, where the coupling 


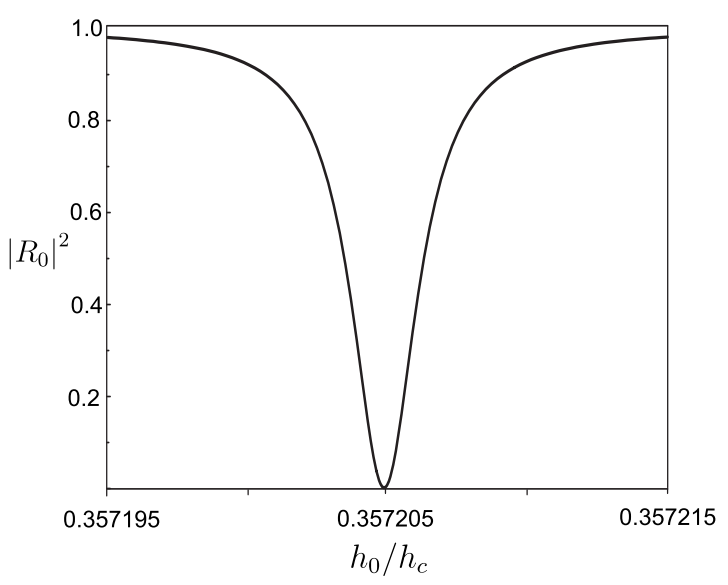

FIG. 10. Numerical simulation of the total suppression of the specular reflection when changing the external magnetic field $h_{0}$ for the forward resonance diffraction $(r=1)$. The calculations were performed for the incident angle $\theta=45^{\circ}$ using Eq. (62) for the harmonic grating with pitch $L=2.75 \times 10^{-4} \mathrm{~mm}$ and modulation magnitude $f_{r}=0.2$. Other parameters used here are: $\nu=1.15 \times 10^{-6}$, $\lambda_{a b}=2.5 \times 10^{-4} \mathrm{~mm}, \lambda_{c} / \lambda_{a b}=7, \varepsilon=4$, and $\Omega=0.95$.

$\left|C_{r}\right| \ll 1$ (when the radiation leakage of the excited SJPWs does not dominate), corresponds to the strongest excitation of the surface waves by the incident waves.

Second, due to the coupling, the specular reflection coefficient, $R_{0}$, differs from the Fresnel coefficient, $R_{F}$, and its modulus becomes less than one. Moreover, as we show below, the reflection of waves with any given frequency $\omega$ $<\omega_{J}$ can be totally suppressed for a certain value of the external magnetic field $h_{0}$ and for the appropriate incident angle $\theta$. This provides a way to control and filter the $\mathrm{THz}$ radiation. Figure 10 shows the total suppression of the reflectivity when changing the external magnetic field. On the other hand, Fig. 11 demonstrates the suppression of the reflectivity when changing the incident angle $\theta$. The numerical calculations were proceeded for the SJPW marked by a solid circle in Fig. 2(a). These are the simplest examples of how we can guide the $\mathrm{THz}$ radiation.

\section{CONCLUSIONS}

Thus, we present a systematic study of the resonance features for the excitation of the SJPWs across the junctions of the layered superconductors. We show the possibility of the

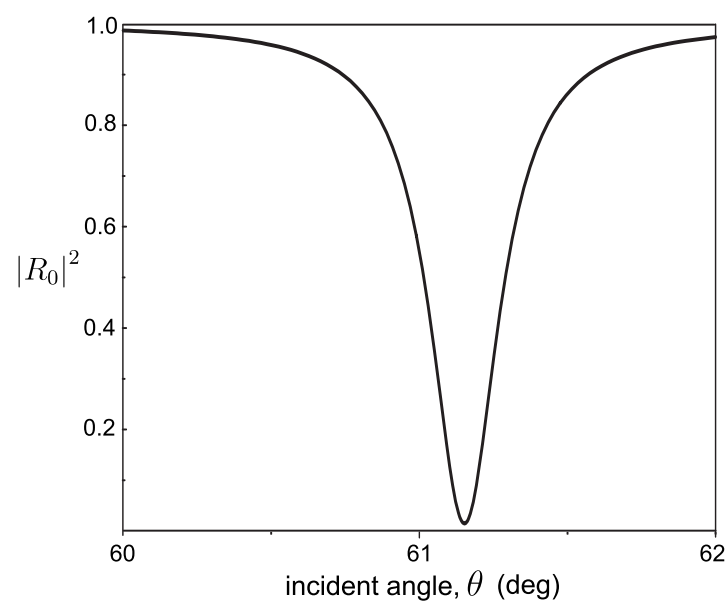

FIG. 11. Numerical simulation of the total suppression of the specular reflection when changing the incident angle $\theta$ for the forward resonance diffraction $(r=1)$. The calculations were performed for the magnetic field $h_{0} / h_{c}=0.357$ using Eq. (62) for the same parameters as in Fig. 10.

SJPW resonance excitation by means of both the ATR and PM methods. The resonance is produced by the excitation of the SJPWs for specific combinations of the incident angle, frequency, and external dc magnetic field. This phenomenon is analogous to the well-known surface-plasmon polariton resonance in the visible and near-infrared region. The analytical study developed here allows us to predict strong resonance effects (total suppression of the specular reflection).

For the PM method, the simplest (in-plane) configuration for a TM-polarized incident wave was examined under single-resonance conditions (i.e., excitation of one running SJPW). The approach used here allows a similar study of the simultaneous excitation of two SJPWs (double resonance), as well as the examination of the so-called "conical diffraction mount" (out-of-plane diffraction). The phenomena discussed here together with nonlinear and quantum effects ${ }^{22}$ in layered superconductors make these materials very promising for different $\mathrm{THz}$ applications.

\section{ACKNOWLEDGMENTS}

D.R.G. and S.S. gratefully acknowledge partial support from EPSRC via Grants No. EP/D072518/1; No. EP/ E042589/1; and No. EP/F005482/1, and ESF network program "AQDJJ."
${ }^{1}$ R. Kleiner, F. Steinmeyer, G. Kunkel, and P. Muller, Phys. Rev. Lett. 68, 2394 (1992).

${ }^{2}$ R. Kleiner and P. Muller, Phys. Rev. B 49, 1327 (1994).

${ }^{3}$ G. Blatter, M. V. Feigel'man, V. B. Geshkenbein, A. I. Larkin, and V. M. Vinokur, Rev. Mod. Phys. 66, 1125 (1994).

${ }^{4}$ E. H. Brandt, Rep. Prog. Phys. 58, 1465 (1995).

${ }^{5}$ V. L. Pokrovsky, Phys. Rep. 288, 325 (1997).

${ }^{6}$ L. N. Bulaevskii, M. P. Maley, and M. Tachiki, Phys. Rev. Lett. 74, 801 (1995); C. C. Homes, T. Timusk, R. Liang, D. A. Bonn, and W. N. Hardy, ibid. 71, 1645 (1993); Y. Matsuda, M. B. Gaifullin, K. Kumagai, K. Kadowaki, T. Mochiku, and K. Hirata, Phys. Rev. B 55, R8685 (1997); K. Kadowaki, I. Kakeya, M. B. Gaifullin, T. Mochiku, S. Takahashi, T. Koyama, and M. Tachiki, ibid. 56, 5617 (1997); I. Iguchi, K. Lee, E. Kume, T. Ishibashi, and K. Sato, ibid. 61, 689 (2000); H. B. Wang, P. H. Wu, and T. Yamashita, Phys. Rev. Lett. 87, 107002 (2001); N. Kameda, M. Tokunaga, T. Tamegai, M. Konczykowski, and S. Okayasu, Phys. Rev. B 69, 180502(R) (2004). 
${ }^{7}$ Y. Matsuda, M. B. Gaifullin, K. Kumagai, K. Kadowaki, and T. Mochiku, Phys. Rev. Lett. 75, 4512 (1995).

${ }^{8}$ S. E. Savel'ev, V. A. Yampol'skii, A. L. Rakhmanov, and F. Nori, arXiv:0903.2969, Rep. Prog. Phys. (to be published).

${ }^{9}$ Y. Tominari, T. Kiwa, H. Murakami, M. Tonouchi, H. Wald, P. Seidel, and H. Schneidewind, Appl. Phys. Lett. 80, 3147 (2002).

${ }^{10}$ J. Zitzmann, A. V. Ustinov, M. Levitchev, and S. Sakai, Phys. Rev. B 66, 064527 (2002).

${ }^{11}$ L. Ozyuzer, A. E. Koshelev, C. Kurter, N. Gopalsami, Q. Li, M. Tachiki, K. Kadowaki, T. Yamamoto, H. Minami, H. Yamaguchi, T. Tachiki, K. E. Gray, W.-K. Kwok, and U. Welp, Science 318, 1291 (2007).

${ }^{12}$ S. Savel'ev, V. Yampol'skii, and F. Nori, Phys. Rev. Lett. 95, 187002 (2005); Physica C 445-448, 183 (2006); V. A. Yampol'skii, A. V. Kats, M. L. Nesterov, A. Yu. Nikitin, T. M. Slipchenko, S. Savel'ev, and F. Nori, Phys. Rev. B 76, 224504 (2007); 79, 214501 (2009).

${ }^{13}$ P. M. Platzman and P. A. Wolff, Waves and Interactions in Solid State Plasmas (Academic, London, 1973).

${ }^{14}$ V. M. Agranovich and D. L. Mills, Surface Polaritons (Nauka, Moscow, 1985); H. Raether, Surface Plasmons (Springer-Verlag, Berlin, 1988); R. Petit, Electromagnetic Theory of Gratings (Springer, New York, 1980).

${ }^{15}$ V. A. Yampol'skii, D. R. Gulevich, S. Savel'ev, and F. Nori, Phys. Rev. B 78, 054502 (2008).
${ }^{16}$ S. Sakai, P. Bodin, and N. F. Pedersen, J. Appl. Phys. 73, 2411 (1993); L. N. Bulaevskii, M. Zamora, D. Baeriswyl, H. Beck, and J. R. Clem, Phys. Rev. B 50, 12831 (1994); S. N. Artemenko and S. V. Remizov, JETP Lett. 66, 853 (1997); Ch. Helm, J. Keller, Ch. Peris, and A. Sergeev, Physica C 362, 43 (2001); Yu. H. Kim and J. Pokharel, ibid. 384, 425 (2003).

${ }^{17}$ S. N. Artemenko and S. V. Remizov, Physica C 362, 200 (2001).

${ }^{18}$ A. E. Koshelev, Phys. Rev. B 62, R3616 (2000).

${ }^{19}$ T. Koyama and M. Tachiki, Phys. Rev. B 54, 16183 (1996).

${ }^{20}$ Ch. Helm and L. N. Bulaevskii, Phys. Rev. B 66, 094514 (2002).

${ }^{21}$ A. V. Kats and V. V. Maslov, Sov. Phys. JETP 35, 264 (1972).

${ }^{22}$ S. Savel'ev, V. Yampol'skii, A. Rakhmanov, and F. Nori, Phys. Rev. B 72, 144515 (2005); S. Savel'ev, A. L. Rakhmanov, and F. Nori, Phys. Rev. Lett. 94, 157004 (2005); S. Savel'ev, A. L. Rakhmanov, V. A. Yampol'skii, and F. Nori, Nat. Phys. 2, 521 (2006); S. Savel'ev, A. L. Rakhmanov, and F. Nori, Phys. Rev. Lett. 98, 077002 (2007); S. Savel'ev, A. L. Rakhmanov, X. Hu, A. Kasumov, and F. Nori, Phys. Rev. B 75, 165417 (2007); D. R. Gulevich, F. V. Kusmartsev, S. Savel'ev, V. A. Yampol'skii, and F. Nori, Phys. Rev. Lett. 101, 127002 (2008); A. L. Rakhmanov, S. E. Savel'ev, and F. Nori, Phys. Rev. B 79, 184504 (2009); L. M. Fisher, I. F. Voloshin, V. S. Gorbachev, S. E. Savel'ev, and V. A. Yampol'skii, Physica C 245, 231 (1995). 